\title{
OSTEOFITOSIS VERTEBRAL EN POBLACIONES PREHISPÁNICAS DE San Pedro de Atacama, norte de Chile
}

\author{
Rocío López-Barrales ${ }^{1,2}$, Mark Hubbe ${ }^{3}$, Eugenio Aspillaga ${ }^{2}$, Walter A. Neves ${ }^{4}$, Hermann \\ M. Niemeyer ${ }^{1}$
}

\section{* Introducción}

\section{Resumen}

Se estudió la osteofitosis vertebral como indicador de estrés físico en grupos humanos prehistóricos de San Pedro de Atacama, durante los períodos Medio (400 DC - 1000 DC) e Intermedio Tardío (1000 DC - 1450 DC). Se registró el grado promedio de osteofitosis vertebral de cada segmento vertebral en 154 individuos adultos de cuatro sitios: Solcor 3, Coyo 3, Quitor 6 y Toconao Oriente. Se comprobó el carácter degenerativo de la osteofitosis vertebral, ya que el grado promedio de osteofitosis fue mayor en el rango etario más viejo. Se observaron diferencias significativas entre sitios sólo en este rango. No se encontraron

diferencias en el grado de osteofitosis entre sexos. Los resultados indicaron que durante el período Intermedio Tardío, tanto hombres como mujeres, sufrieron un estrés físico sobre la espalda menor que las poblaciones que vivieron durante el período Medio, especialmente a finales de este período, posiblemente por cambios en los patrones de actividad, ya sea en la energía invertida en sus actividades y por consecuencia, en la intensidad de las actividades físicas desarrolladas, o en diferencias en el tipo de actividades desarrolladas.

Palabras claves: bioarqueología - estrés físico - período Medio - período Intermedio Tardío.

\begin{abstract}
Vertebral osteophytosis was studied as a marker for physical stress in prehistoric populations of San Pedro de Atacama from the Middle (400 - 1.000 A.D.) and Late Intermediate (1.000 - 1.450 A.D.) periods. The mean degree of osteophytosis was recorded in each vertebral segment of 154 individuals from four sites: Solcor 3, Coyo 3, Quitor 6 and Toconao Oriente. Osteophytosis prevalence was more severe in older individuals, corroborating its degenerative nature. However, after correction for age impact in osteophytosis prevalence, significant differences were observed between sites. Differences in osteophytosis prevalence between sexes were not significant. The results show that during the Late Intermediate period populations of both sexes suffered less vertebral stress than during the Middle period, particularly

during the its end. Changes in activity patterns (amount of energy invested, intensity or type of activities undertaken) could account for the differences found.
\end{abstract}

Key words: bioarchaeology - physical stress - Middle period - Late Intermediate period.

Recibido: Junio 2014. Aceptado: Febrero 2015
San Pedro de Atacama está constituido por un conjunto de pequeños oasis situados al norte del salar de Atacama en la confluencia de los ríos San Pedro y Vilama, en la Región de Antofagasta del norte de Chile, a una altitud de 2500 msnm (Figura 1). Ha tenido un desarrollo cultural continuo desde por lo menos los primeros asentamientos estables alrededor del $500 \mathrm{AC}$ hasta la actualidad, marcado por períodos que mostraron variaciones importantes en las conexiones que mantuvo con otras regiones de los Andes Centro-Sur (Le Paige 1965; Núñez 2007).

Gracias a la excelente conservación de restos orgánicos en el área, se han podido registrar extensos cementerios que albergan restos humanos de gran parte de su prehistoria agroalfarera, lo que ha permitido conocer diferentes aspectos de la vida de estas poblaciones. En esta línea se encuentra una serie de estudios centrados en calidad y estilo de vida, los que han demostrado diferencias importantes entre distintos períodos, especialmente entre los períodos Medio (400-100o DC) e Intermedio Tardío (1000 DC - 1450 DC). Estos períodos presentan características culturales y económicas distintivas, por lo que la transición del período Medio al Intermedio Tardío trajo consigo no solo cambios culturales, sino también cambios en el estilo de vida y en la salud de las poblaciones. La mayor parte de los estudios que comparan ambos períodos están centrados en cambios de dieta y salud (Costa et al. 1998, 2004; Lessa y Mendoça de Souza 2004; Torres-Rouff y Costa 2006; Torres-Rouff 2011; Tótora da Glória et al. 2011; Hubbe et al. 2012); en consecuencia, es deseable desarrollar otros estudios más específicos en torno a las actividades físicas desarrolladas por las poblaciones y a sus efectos en las condiciones de salud de éstas.

1 Departamento de Ciencias Ecológicas, Facultad de Ciencias, Universidad de Chile. Av. Capitán Ignacio Carrera Pinto No 1045 , Nuñoa, Santiago, CHILE.Email: rociolopez@ug.uchile.clyniemeyer@abulafia.ciencias.uchile.cl 


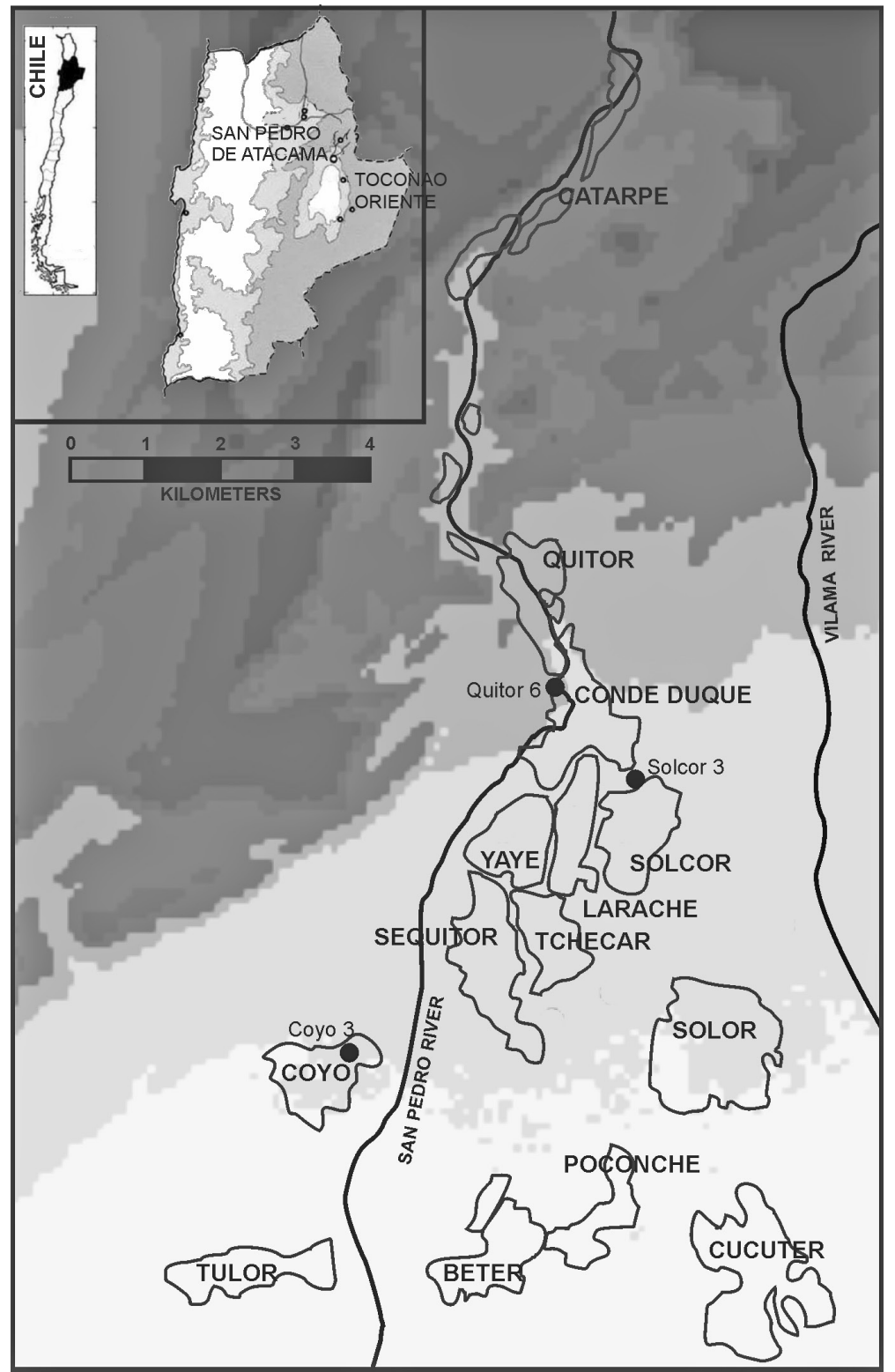

Figura 1. Localización de los sitios analizados en este estudio.

2 Departamento de Antropología, Facultad de Ciencias Sociales, Universidad de Chile. Av. Capitán Ignacio Carrera Pinto $\mathrm{N}^{\circ} 1045$, Ññoa, Santiago, CHILE.Email: rociolopez@ug.uchile.clyeaspilla@uchile.cl

3 Department of Anthropology, The Ohio State University. Columbus, $\mathrm{OH}$ 43210, USA. Instituto de Investigaciones Arqueológicas y Museo R.P. Gustavo Le Paige S.J., Universidad Católica del Norte. Gustavo Le Paige 380, San Pedro de Atacama, CHILE. Email: mhubbe@ucn.cl

4 Laboratório de Estudos Evolutivos Humanos, Departamento de Genética e Biologia Evolutiva, Universidade de São Paulo. Edifício André Dreyfus, Rua do Matão, 277, Sao Paulo, BRASIL. Email: waneves@ib.usp.br
Este artículo estudia la osteofitosis vertebral como indicador de estrés físico en grupos humanos prehistóricos de San Pedro de Atacama, durante los períodos Medio e Intermedio Tardío, con el objeto de evaluar posibles cambios en las actividades físicas, o en la intensidad de éstas, y relacionarlos con los cambios sociales y económicos que se han planteado para estos dos períodos, complementando así la escasa información en torno al impacto de las actividades desarrolladas por estas poblaciones en su biología. 


\section{Contexto cronológico cultural}

El período Medio en San Pedro de Atacama se caracteriza por un gran desarrollo cultural y económico, reflejado en la calidad y cantidad de las ofrendas funerarias, en el aumento de la densidad de los cementerios y en el aumento de las interacciones con otras regiones (Berenguer y Dauelsberg 1989; Núñez 2007; Torres-Rouff et al. 2013). Dicho auge trajo consigo una mejora en la calidad de vida de las poblaciones, particularmente en aspectos nutricionales (Neves y Costa 1998; Costa et al. 2004; Hubbe et al. 2012). La participación de San Pedro de Atacama en una amplia red de interacción regional le permitió un contacto con distintas zonas del área Centro-Sur andina, incluyendo Tiwanaku, del cual recibió una gran influencia (Berenguer y Dauelsberg 1989; Núñez 2007). Se observa también un aumento de la complejidad social relacionado con la influencia de Tiwanaku y reflejado en diferencias en la calidad y diversidad del ajuar funerario de las tumbas de este período (Núñez 2007). Estas diferencias también se traducen en diferencias en la dieta (Hubbe et al. 2012) y en el patrón de traumas craneales (Torres-Rouff 2011). Solo una parte de la población tuvo un mayor acceso a fuentes proteicas, en particular individuos masculinos con un mayor estatus social (Hubbe et al. 2012). Asimismo, estos individuos presentan menor frecuencia de traumas craneales, lo que indicaría tensiones al interior de la sociedad atacameña relacionadas con las diferencias sociales sugeridas por el registro arqueológico (Torres-Rouff 2011).

El período Intermedio Tardío se inicia con la pérdida de los vínculos con Tiwanaku y con ello la integración regional alcanzada en el período Medio (Uribe 2002; Núñez 2007). Sumado probablemente a la crisis ambiental a la que se vio sometida una parte importante de Sudaméri$\mathrm{ca}$, se da inicio a un período de consolidación de desarrollos regionales con nuevas relaciones con el altiplano meridional (Schiappacasse et al. 1989; Uribe 2002; Uribe et al. 2004). Esto trajo consigo una serie de cambios en los oasis atacameños, tanto culturales como en la calidad y estilo de vida de las poblaciones: i) se observan cambios en el patrón funerario, con nuevas formas de enterramiento y con un supuesto "empobrecimiento" de las ofrendas en relación con la riqueza del período anterior, ii) aparece un nuevo patrón de asentamiento con un énfasis en las quebradas, iii) se construyen pucarás, recin- tos fortificados que sugieren la aparición de conflictos sociales (Schiappacasse et al. 1989; Uribe et al. 2004; Núñez 2007), lo que es apoyado por estudios de traumas craneales que muestran que la región tuvo un grado mayor de violencia (Lessa y Mendoça de Souza 2004; Torres-Rouff y Costa 2006), iv) también se produce una disminución de los niveles de salud, reflejados en una menor estatura y el aumento de caries y de hipoplasias del esmalte que indicarían una disminución del acceso a proteína animal, así como una mayor exposición a eventos de estrés (Costa et al. 2004), y un aumento de enfermedades infecciosas no específicas (Tótora da Glória et al. 2011).

En relación al uso del cuerpo y el estrés biomecánico, existen pocos estudios. Uno de ellos, sobre la prevalencia de traumas postcraneales en algunos sitios de San Pedro de Atacama, no muestra cambios significativos entre los distintos períodos de su prehistoria agroalfarera, lo que sugiere que los riesgos asociados a las actividades físicas y, por extensión, las actividades cotidianas desarrolladas por las poblaciones, no variaron mayormente a lo largo del tiempo (Costa et al. 1998, 2004). Lessa y Mendonça de Souza (2003), en cambio, observaron diferencias en la prevalencia de traumatismos cotidianos en el sitio Solcor 3 , entre un grupo anterior al período de influencia de Tiwanaku y otro en los momentos de su mayor influencia.

Estas diferencias hacen concluir a estas investigadoras que las actividades relacionadas con el intercambio a larga distancia (tales como cargar y transportar) pudieron haberse intensificado durante el período Medio. Un estudio llevado a cabo por Pomeroy (2013), que evalúa la robusticidad de los miembros inferiores entre diversas muestras de San Pedro de Atacama y de otros lugares del norte de Chile encuentra que las muestras de los momentos finales del período Medio y la transición hacia el Intermedio Tardío en San Pedro de Atacama presentan mayor robusticidad que las muestras del momento de apogeo del período Medio. Pomeroy sostiene que estos resultados apoyan la hipótesis de que durante el período Medio San Pedro de Atacama era parte de redes de intercambio a larga distancia manejadas por Tiwanaku, y que con el colapso de Tiwanaku las poblaciones de San Pedro de Atacama debieron participar de manera más activa en este tráfico, aunque no descarta 
otras explicaciones como cambios en actividades productivas.

\section{Osteofitosis vertebral}

Las actividades habituales u ocupacionales pueden ejercer un estrés biomecánico continuo y prolongado, lo que a su vez puede provocar el desarrollo de alteraciones óseas y dentales (Kennedy 1989). La osteoartritis y la osteofitosis vertebral son marcadores utilizados tradicionalmente en estudios bioarqueológicos orientados a inferir el comportamiento habitual de poblaciones del pasado.

La osteoartritis o enfermedad degenerativa articular es una de las condiciones patológicas más comunes en el ser humano (Bridges 1992, 1994; Jurmain y Kilgore 1995). En la columna vertebral se produce una condición relacionada con la osteoartritis que ha sido denominada osteofitosis vertebral (Jurmain y Kilgore 1995). Esta se caracteriza por el crecimiento óseo en el margen de los cuerpos vertebrales, que puede conducir a la fusión entre vértebras adyacentes en los casos más severos (Kellgren y Lawrence 1958; Bridges 1994; Jurmain y Kilgore 1995; Knüsel et al. 1997; Ortner 2003; Van Der Merwe et al. 2006). Se considera una característica concomitante a la degeneración del disco intervertebral (Kalichman 2010; Adams 2012), aunque también se ha registrado su aparición de manera independiente (Oishi et al. 2003).

Estudios en poblaciones modernas han relacionado la degeneración del disco intervertebral con actividades físicas intensas, lo que permite plantear que el factor mecánico es importante en la patogénesis de esta condición (Kellgren y Lawrence 1955, 1958; O'Neill et al. 1999; Schmitt et al. 2004). Al ser una de las alteraciones osteológicas que con mayor frecuencia se observan en los restos esqueletales, la osteofitosis vertebral ha sido el foco de numerosas investigaciones bioantropológicas. Diversos estudios han abordado el rol de la carga mecánica repetitiva sobre la columna vertebral, planteando que ciertas actividades específicas pueden tener un efecto importante sobre el patrón e intensidad de la osteofitosis en la columna (Bridges 1992, 1994; Sofaer Derevenski 2000), lo que permitiría identificar patrones de actividad e inferir así el modo de vida de las poblaciones del pasado.

Sin embargo, algunas investigaciones de carácter biomé- dico y epidemiológico han criticado el uso de la osteofitosis como indicador de estrés ocupacional aduciendo que se trataría de una condición en la cual participarían distintos factores, enfatizando su alta correlación con la edad y la influencia de la propia posición bípeda del ser humano y concluyendo que la columna vertebral no parecería ser una estructura ideal para estudiar marcadores de estrés ocupacional (Knüsel et al. 1997). Además, algunas investigaciones en poblaciones modernas centradas en la degeneración del disco intervertebral han sugerido que los factores genéticos y hereditarios juegan un rol importante (Battié et al. 2009; Kalichman 2010).

La diversidad de resultados de estas investigaciones sugiere que ambos factores, el genético y el mecánico, son importantes en la degeneración del disco intervertebral (Adams et al. 2009; Adams 2012), proceso que posteriormente da lugar a la formación de osteofitos (Kumaresan et al. 2001). En consecuencia, aunque quizás no se pueda llegar a conocer el tipo de actividades específicas que originan la patología (Jurmain 1990), este tipo de estudios entrega información acerca de la intensidad del estrés aplicado a esta estructura anatómica producto de distintos factores, incluyendo el cultural, reflejado en las actividades físicas y/o productivas —en su sentido más amplio - realizadas por un grupo humano en particular.

\section{* Materiales y métodos}

El estudio se realizó en individuos adultos procedentes de cuatro sitios en el salar de Atacama: Toconao Oriente, Solcor 3, Coyo 3 y Quitor 6, los que se ubican cronológica y culturalmente en los períodos Medio e Intermedio Tardío (Tabla 1). Los cuatro sitios corresponden a cementerios, o a una parte de ellos, que fueron excavados durante las décadas de 1980 y 1990 por el equipo formado por los investigadores Agustín Llagostera y María Antonieta Costa. Tres de ellos se ubican en San Pedro de Atacama y uno de ellos, Toconao Oriente, en la localidad de Toconao, a $38 \mathrm{~km}$ al sur (véase Figura 1).

Solcor 3 corresponde a un cementerio compuesto por 93 tumbas con 153 cuerpos (Bravo y Llagostera 1986). Las fechas obtenidas para este sitio indican que fue utilizado durante el período Medio (Llagostera et al. 1988; Hubbe et al. 2012). La importante presencia de ofrendas con iconografía Tiwanaku (entre un $15 \%$ y un $20 \%$ de las 


\begin{tabular}{|c|c|c|c|c|c|c|c|c|}
\hline \multirow{2}{*}{ Período } & \multirow{2}{*}{ Sitio } & \multicolumn{3}{|c|}{ Femenino } & \multicolumn{3}{|c|}{ Masculino } & \multirow{2}{*}{ Tota } \\
\hline & & $(20-34)$ & $(35-49)$ & $(>50)$ & $(20-34)$ & $(35-49)$ & $(>50)$ & \\
\hline \multirow{3}{*}{ Medio } & Solcor 3 Elite & 11 & 9 & o & 9 & 6 & o & 35 \\
\hline & Solcor 3 No Elite & 3 & 8 & 1 & 8 & 6 & o & 26 \\
\hline & (Total & 14 & 17 & 1 & 17 & 12 & o & 61) \\
\hline Finales Medio & Coyo 3 & 5 & 15 & 2 & 6 & 17 & 1 & 46 \\
\hline \multirow{3}{*}{ Intermedio Tardío } & Quitor 6 & 14 & 4 & 2 & 11 & o & o & 31 \\
\hline & Toconao Oriente & 2 & 6 & ○ & 2 & 6 & o & 16 \\
\hline & Total & 35 & 42 & 5 & 36 & 35 & 1 & 154 \\
\hline
\end{tabular}

Tabla 1. Muestras óseas para el análisis de osteofitosis vertebral.

tumbas) y de otras zonas, y la gran cantidad de ofrendas locales sugiere la presencia de un grupo de estatus social más alto al interior de este cementerio (Bravo y Llagostera 1986; Torres-Rouff 2008, 2011). Bravo y Llagostera (1986) constataron la existencia de sectores al interior del cementerio definidos por patrones de asociación del ajuar encontrado junto a los cuerpos, lo que permitió a Llagostera et al. (1988) plantear la presencia de dos grupos, uno previo al contacto con Tiwanaku y otro en pleno contacto con la entidad altiplánica. Sin embargo, la recalibración y la toma de nuevos fechados han demostrado que los grupos observados por Bravo y Llagostera (1986) se tratarían de dos grupos sincrónicos, posiblemente reflejando distinto estatus social (Torres-Rouff y Hubbe 2013).

Coyo 3 corresponde a un amplio cementerio, parcialmente excavado, ubicado cronológicamente hacia finales del período Medio (Costa y Llagostera 1994). En él se encontraron 51 tumbas con 80 individuos, con ofrendas que según los autores mencionados disminuyeron tanto en calidad como en cantidad, respecto a otros sitios del mismo período, aunque se sigue observando alguna influencia de Tiwanaku.

Quitor 6 es un extenso cementerio que fue excavado principalmente por Le Paige, cuyo sector norte fue posteriormente excavado por Agustín Llagostera, quien halló 42 tumbas con 33 adultos (Costa 1988). Este sector corresponde a una fase tardía de ocupación en relación al resto del cementerio, el cual fue ocupado durante el período Medio. Las fechas del sector norte lo ubican en el período Intermedio Tardío y no se evidencia interacción con Tiwanaku. En comparación con el resto del cemen- terio, este sector presenta escasas ofrendas o ausencia de ellas (Costa 1988).

Toconao Oriente es un gran cementerio que fue ocupado desde el período Formativo (Berenguer et al. 1988); la muestra utilizada en este estudio pertenece a un sector excavado por María Antonieta Costa y Agustín Llagostera que arrojó fechas pertenecientes al período Intermedio Tardío (Torres-Rouff et al. 2013). La información sobre su excavación no ha sido publicada.

Se observó y registró el grado promedio de osteofitosis vertebral en 154 individuos adultos. La estimación de sexo y edad de estos individuos fue realizada bajo los estándares compendiados y propuestos por Ubelaker y Buikstra (1994). La estimación de sexo se basó en indicadores del cráneo y coxales. Para la estimación de edad de muerte se utilizaron distintos métodos, entre ellos, suturas craneales, sínfisis púbica, superficie auricular del ilion y centros de osificación secundarios. Para efecto de este estudio y en consideración a la correlación entre osteofitosis vertebral y edad detectada en otras investigaciones, se seleccionaron los esqueletos de individuos en los que la edad estimada superara los 20 años, debido a que hay una mayor probabilidad de observar este fenómeno en adultos. Esto significó que se consideraran tres categorías etarias: entre 20 y 34 años, entre 35 y 49 años y más de 50 años.

Se evaluó y registró la osteofitosis en los márgenes de las caras superior e inferior de los cuerpos vertebrales por separado. En el caso de las vértebras cervicales la observación se realizó a partir de $C_{3}$, dejando fuera del análisis 


\begin{tabular}{|l|l|}
\hline Grados & Características \\
\hline 0 & Ausencia de rasgos degenerativos. \\
\hline 1 (leve) & Formación de labiamiento en partes discretas del margen. \\
\hline 2 (moderado) & $\begin{array}{l}\text { Presencia de un labiamiento cuya sumatoria ocupa como } \\
\text { mínimo la mitad de la extensión del margen. Pueden } \\
\text { aparecer osteofitos discretos asociados. }\end{array}$ \\
\hline 3 (acentuado) & $\begin{array}{l}\text { Presencia generalizada de osteofitos horizontales en el } \\
\text { margen de los cuerpos y/o aserramiento del labio. }\end{array}$ \\
\hline 4 (severo) & $\begin{array}{l}\text { Presencia de osteofitos muy largos, verticales, articulados } \\
\text { y/o anquilosados. }\end{array}$ \\
\hline
\end{tabular}

Tabla 2. Grados utilizados en la observación y registro de osteofitosis vertebral en las caras superior e inferior de los cuerpos vertebrales.

el atlas y el axis de todos los individuos. Esto dio un total de 44 observaciones por individuo en el caso de columnas completas (10 observaciones en el segmento cervical, 24 en el torácico y 10 en el lumbar). El modo de registro consistió en la observación del grado de desarrollo de osteofitos en los márgenes vertebrales, método de observación utilizado por el laboratorio de Estudios Evolutivos Humanos de la Universidad de Sao Paulo (Tabla 2) y adaptado de los trabajos de Jurmain (1990).

Para el análisis estadístico se utilizó el promedio del grado de osteofitosis de cada segmento vertebral por individuo. Dado que existe una relación entre la prevalencia de rasgos degenerativos de las vértebras y el peso soportado se dividió la columna torácica en dos, un segmento torácico superior ( $\mathrm{T} 1$ - T6) y un segmento torácico inferior ( $\left.\mathrm{T}_{7}-\mathrm{T}_{12}\right)$, lo que dio lugar a cuatro segmentos por individuo (cervical, torácico superior, torácico inferior y lumbar).

La mayor parte de las investigaciones publicadas sobre osteofitosis se han centrado en el porcentaje de individuos o de vértebras de cada segmento vertebral con la condición de osteofitosis (prevalencia), o con los estados más severos de ella (severidad) (Bridges 1994; O’Neill et al. 1999; Sofaer Derevenski 2000; Rojas-Sepúlveda et al. 2008). El uso del valor promedio por segmento evita el riesgo de sobreestimación ya que, en el caso de considerar el número de individuos con osteofitos por lo menos en una de las vértebras, una sola vértebra afectada podría sobreestimar la medición de ese individuo.
Para evitar sesgos en relación con el grado de conservación de las estructuras estudiadas, el registro se realizó en las vértebras que no presentaran pérdida de tejido óseo o cambios en la morfología debido a efectos tafonómicos; si presentaban tales defectos, éstos no debían superar un tercio del margen de cada cara (superior e inferior) de los cuerpos vertebrales. Además se eliminaron los segmentos vertebrales que no permitieran registrar, al menos, un $50 \%$ de las observaciones posibles. Esta eliminación parcial permitió considerar a los individuos que no tenían información para toda la columna vertebral. En la Figura 2 se presenta la proporción de vértebras conservadas posibles de analizar por lo menos en una de sus caras.

Para el análisis comparado de la osteofitosis vertebral se llevó a cabo un ANOVA factorial mediante un modelo lineal general (GLM en inglés). Una de las ventajas de este análisis es que permite considerar variables de distinta naturaleza, ya sean discretas o continuas, así como buscar la contribución independiente de cada factor (variables independientes o explicativas) sobre la variable

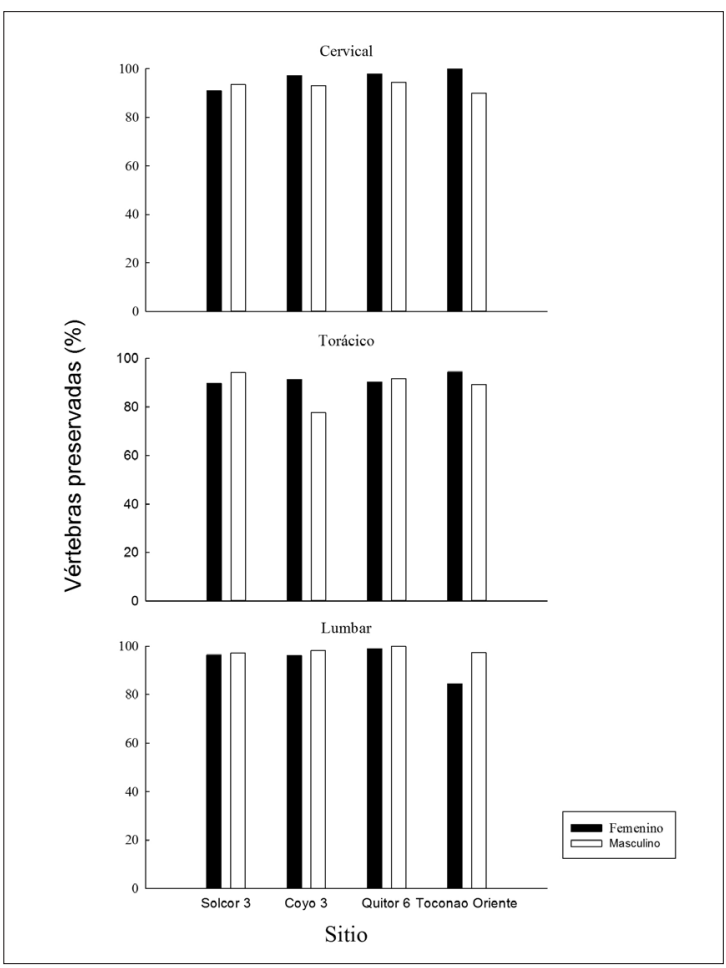

Figura 2. Completitud de las columnas vertebrales. Las barras indican el porcentaje de vértebras observadas en cada sitio. Las barras de color negro corresponden a los individuos femeninos y las blancas a los masculinos. 
dependiente (o respuesta) (Hair et al. 2009). Se desarrolló un modelo en el que la osteofitosis vertebral, como variable respuesta, se contrastara con los factores edad, sexo y sitio, este último relacionado directamente con el período cultural en el presente estudio.

Para la construcción del modelo lineal general se excluyeron los individuos de la categoría 3 de edad, es decir, mayores de 50 años, ya que al solo contar con seis individuos en esta categoría el modelo perdía robustez. Por otra parte, los individuos estudiados del sitio Solcor 3 fueron asignados, previo a la construcción del modelo general, a dos subgrupos que diferían en estatus social, definidos siguiendo la propuesta de Hubbe et al. (2012) y sobre la base de los estudios llevados a cabo por Bravo y Llagostera (1986) donde se sugiere la presencia de estos dos grupos. El grupo "élite" se caracteriza por tener en su ajuar funerario objetos Tiwanaku y cerámica Negra Pulida; el grupo "no élite" por la ausencia de elementos Tiwanaku y tener cerámica más tosca (Bravo y Llagostera 1986; Llagostera et al. 1988; Torres-Rouff y Hubbe 2013). Dado que no se

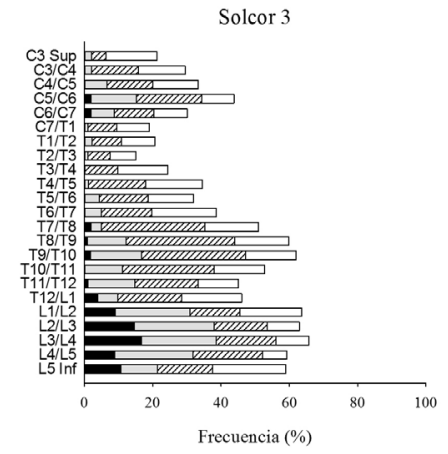

Quitor 6

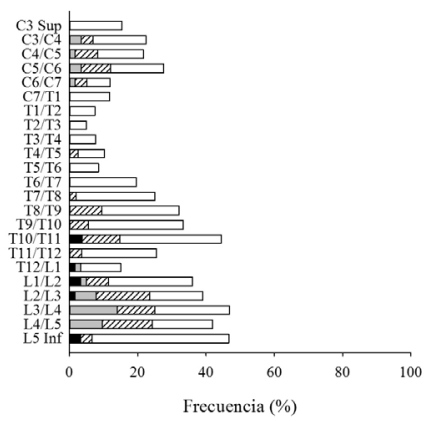

encontraron diferencias significativas en ninguno de los segmentos vertebrales (prueba de Mann-Whitney; $\mathrm{P}>0,05)$ cuando se compararon ambos grupos, éstos fueron agrupados en el análisis que utilizó el modelo lineal general.

Una vez verificada mediante el modelo lineal general la influencia de alguno de los factores sobre la osteofitosis, se realizaron comparaciones post hoc (a posteriori) entre los sitios para identificar cuáles se diferenciaban significativamente entre sí. En el caso de este estudio se utilizó la prueba HSD de Tukey para grupos desiguales, es decir, con diferente número de repeticiones por tratamiento (unequal N HSD en inglés) debido a las diferencias de tamaño entre las series esqueletales. Los análisis se realizaron en el programa Statistica 7 (Statsoft, Inc.).

\section{$*$ Resultados}

La Figura 3 muestra la prevalencia de osteofitosis en sus distintos grados para cada articulación a lo largo de la
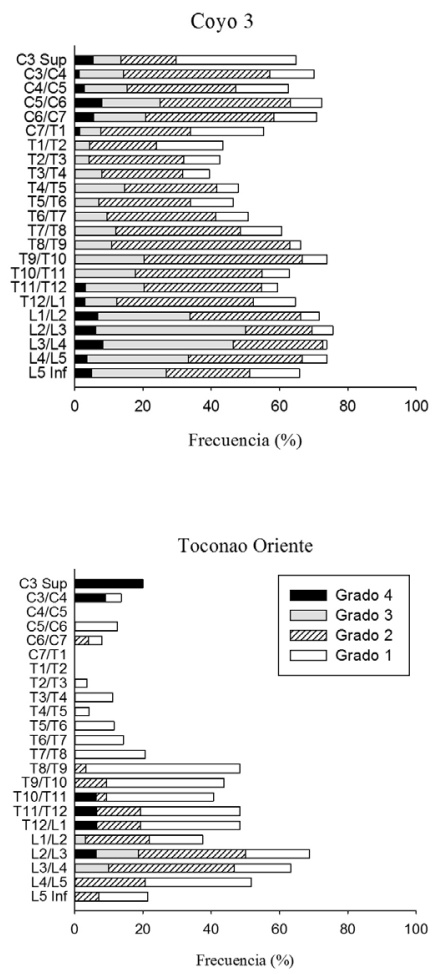

Figura 3. Prevalencia de la osteofitosis a lo largo de la columna vertebral. Las barras apiladas indican la frecuencia de cada grado promedio de osteofitosis vertebral en cada articulación desde $\mathrm{C}_{3}$ superior hasta $\mathrm{L}_{5}$ inferior. El complemento de las barras que falta para llegar a $100 \%$ corresponde a articulaciones con grado o (ausencia de osteofitosis). 
columna vertebral. En esta figura se aprecia que la osteofitosis se presenta con mayor frecuencia a lo largo de la columna vertebral en los sitios del período Medio, ya sea en su momento de mayor apogeo (Solcor 3 ) o hacia finales del mismo (Coyo 3), y que la región lumbar aparecería con mayor prevalencia en todos los cementerios seguida de los segmentos torácico inferior, cervical y torácico superior.

Las articulaciones que presentan una mayor frecuencia de osteofitosis en el segmento cervical son la cara superior de $\mathrm{C}_{3}$ en Toconao Oriente y en $\mathrm{C}_{5}$ - C6 para los otros sitios, en el segmento torácico T8 - T9 en Toconao Oriente, T9-Tio en Solcor 3 y Coyo 3 y Tro-Til en Quitor 6 y en el segmento lumbar L2/L3 en Coyo 3 y Toconao Oriente y L3/L4 en Solcor 3 y Quitor 6. Estos loci coincidirían con los puntos de máxima curvatura de la columna para los segmentos cervical ( $\left.\mathrm{C}_{3}-\mathrm{C}_{5}\right)$ y lumbar ( $\mathrm{L}_{3}$ y $\left.\mathrm{L}_{4}\right)$, como ya lo han constatado otras investigaciones (Nathan 1962; Van der Merwe et al. 2006; Kim et al. 2012), a diferencia del segmento torácico donde la mayor prevalencia de osteofitosis se da por debajo del punto de máxima curvatura, T5 - T8. La (Tabla 3) muestra el grado promedio de osteofitosis vertebral por segmento, sitio y rango etario de los individuos analizados. El grado promedio de los sitios varió entre 0,15 y 0,97 en el segmento cervical, entre 0,04 y 0,62 en el torácico superior, entre 0,46 y 1,02 en el torácico inferior y entre 0,86 y 1,43 en el lumbar.

Coyo 3 presentó el mayor grado promedio de osteofitosis en los segmentos cervical y torácico superior, y no muy distinto del de Solcor 3, el sitio con mayor promedio en los segmentos torácico inferior y lumbar. Por otra parte, los grados promedio fueron considerablemente menores en los sitios Toconao Oriente y Quitor 6, ambos del período Intermedio Tardío.

Al ejecutar el modelo lineal general bajo las condiciones descritas en la sección Materiales y métodos, se observó que (Tabla 4) solo las variables edad y sitio - de manera independiente y la interacción entre ambas - afectaron significativamente $(\mathrm{P}<0,05)$ la expresión de osteofitosis vertebral en toda la columna, con excepción del segmento torácico inferior ( $\mathrm{T}_{7}-\mathrm{T}_{12}$ ) donde solo influyeron estas variables de manera independiente. En todos los casos, las interacciones significativas entre sitio y edad son ordinales (es decir, la interacción no cambia el orden relativo de los factores principales, sitio y edad; véase Figura 4), lo que permite la interpretación independiente de los factores principales (Hair et al. 2009). La variable sexo no mostró significancia estadística para ninguno de los segmentos vertebrales, por lo que en los análisis posteriores se agruparon los datos para ambos sexos.

Cuando se evaluó la interacción entre el sitio y la edad, se observó que las diferencias en grado de osteofitosis entre los distintos sitios eran mediadas por el rango etario de los individuos analizados, encontrándose una interacción ordinal entre ambas variables (Figura 4), es decir, la influencia de la edad no fue igual entre los distintos sitios, pero mostró la misma dirección (el rango etario entre 35 a 49 años mostró siempre mayor grado de osteofitosis que el rango entre 20 y 34 años). Esto resulta relevante ya que las diferencias solo se observaron en la edad mayor, confirmando el carácter degenerativo de la condición.

En los análisis a posteriori se realizaron comparaciones entre sitios por cada rango etario, agrupando los datos para ambos sexos. En los individuos cuya edad se encontraba entre los 20 y 34 años no se encontraron diferencias significativas (datos no mostrados), en tanto que en el grupo etario 35 - 49 años se encontraron diferencias significativas $(\mathrm{P}<\mathrm{O}, \mathrm{Ol})$ entre los sitios en todos los segmentos vertebrales (Tabla 5).

En el segmento cervical se observó que Coyo 3 presentaba el mayor grado de osteofitosis (Figura 4); las diferencias fueron significativas cuando se comparó con Solcor 3 y Toconao Oriente, y marginalmente no significativa cuando se comparó con Quitor 6. En los segmentos torácico y lumbar, los grados promedio de osteofitosis en Solcor 3 y Coyo 3 fueron más altos que en Quitor 6 y Toconao Oriente, aunque las diferencias fueron significativas entre Coyo 3 y Toconao Oriente para todos los segmentos vertebrales y solo entre Solcor 3 y Toconao Oriente en los segmentos torácico inferior y lumbar. Entre los sitios Quitor 6 y Toconao Oriente no existieron diferencias significativas a lo largo de toda la columna (Tabla 5).

\section{* Discusión}

Como fue expuesto en los antecedentes, la osteofitosis vertebral es una condición multifactorial, donde genes, 


\begin{tabular}{|c|c|c|c|c|c|c|}
\hline \multicolumn{7}{|l|}{ a. Cervical } \\
\hline \multirow{2}{*}{ Período } & \multirow{2}{*}{ Sitio } & \multicolumn{2}{|c|}{ Femenino } & \multicolumn{2}{|c|}{ Masculino } & \multirow{2}{*}{ Promedio } \\
\hline & & $20-34$ & $35-49$ & $20-34$ & $35-49$ & \\
\hline Medio & Solcor 3 & 0,233 & 1,219 & 0,159 & 0,611 & 0,555 \\
\hline Finales Medio & Coyo 3 & 0,340 & 1,726 & 0,222 & 1,573 & 0,965 \\
\hline \multirow{2}{*}{ Intermedio Tardío } & Quitor 6 & 0,055 & 0,350 & 0,461 & - & 0,289 \\
\hline & Toconao Oriente & 0,200 & 0,265 & 0,000 & 0,120 & 0,146 \\
\hline \multicolumn{4}{|c|}{ b. Torácico superior $\left(\mathrm{T}_{1}-\mathrm{T} 6\right)$} & & & \\
\hline \multirow{2}{*}{ Período } & \multirow{2}{*}{ Sitio } & \multicolumn{2}{|c|}{ Femenino } & \multicolumn{2}{|c|}{ Masculino } & \multirow{2}{*}{ Promedio } \\
\hline & & $20-34$ & $35-49$ & $20-34$ & $35-49$ & \\
\hline Medio & Solcor 3 & 0,116 & 0,707 & 0,119 & 0,569 & 0,377 \\
\hline Finales Medio & Coyo 3 & 0,208 & 1,333 & 0,000 & 0,939 & 0,620 \\
\hline \multirow{2}{*}{ Intermedio Tardío } & Quitor 6 & 0,077 & 0,227 & 0,093 & - & 0,133 \\
\hline & Toconao Oriente & 0,000 & 0,090 & 0,050 & 0,021 & 0,040 \\
\hline \multicolumn{7}{|c|}{ c. Torácico inferior $\left(\mathrm{T}_{7}-\mathrm{T}_{12}\right)$} \\
\hline \multirow{2}{*}{ Período } & \multirow{2}{*}{ Sitio } & \multicolumn{2}{|c|}{ Femenino } & \multicolumn{2}{|c|}{ Masculino } & \multirow{2}{*}{ Promedio } \\
\hline & & $20-34$ & $35-49$ & $20-34$ & $35-49$ & \\
\hline Medio & Solcor 3 & 0,912 & 1,618 & 0,321 & 1,231 & 1,021 \\
\hline Finales Medio & $\mathrm{Coyo}_{3}$ & 0,521 & 1,766 & 0,405 & 1,384 & 1,019 \\
\hline \multirow{2}{*}{ Intermedio Tardío } & Quitor 6 & 0,288 & 0,750 & 0,333 & - & 0,457 \\
\hline & Toconao Oriente & 0,194 & 0,542 & 0,333 & 0,773 & 0,461 \\
\hline \multicolumn{7}{|l|}{ d. Lumbar } \\
\hline \multirow{2}{*}{ Período } & \multirow{2}{*}{ Sitio } & \multicolumn{2}{|c|}{ Femenino } & \multicolumn{2}{|c|}{ Masculino } & \multirow{2}{*}{ Promedio } \\
\hline & & $20-34$ & $35-49$ & $20-34$ & $35-49$ & \\
\hline Medio & Solcor 3 & 0.819 & 2.426 & 0.692 & 2.01 & 1.487 \\
\hline Finales Medio & Coyo 3 & 0,863 & 2,131 & 0,46 & 2,254 & 1,427 \\
\hline \multirow{2}{*}{ Intermedio Tardío } & Quitor 6 & 0,396 & 1,278 & 0,911 & - & 0,862 \\
\hline & Toconao Oriente & 0,65 & 1,173 & 0,8 & 0,846 & 0,867 \\
\hline
\end{tabular}

Tabla 3. Grado de osteofitosis vertebral de los sitios analizados diferenciados en sexo y edad por cada segmento vertebral.

edad y carga física afectan su aparición y severidad. Los datos obtenidos permitieron verificar la relación entre grado de osteofitosis y edad, establecida ya por diversos autores, mostrando así la importancia de su carácter degenerativo. Es así como el grupo etario más joven (20 - 34 años) mostró un grado promedio bajo de osteofitosis vertebral en cada sitio y segmento vertebral, lo que es esperable ya que la aparición de esta condición se da generalmente en la adultez media. Esto también indicaría el carácter acumulativo de esta condición.
La Figura 4 sugiere que en el grupo etario más viejo ( 35 - 49 años) las muestras del período Intermedio Tardío (Quitor 6 y Toconao Oriente) muestran un grado de osteofitosis vertebral menor que las del período Medio (Solcor 3) y que las de la transición hacia el período Intermedio Tardío (Coyo 3); sin embargo, el efecto fue significativo en todos los segmentos entre Toconao Oriente y Coyo 3, y solo en los segmentos inferiores entre Toconao Oriente y Solcor 3 (Tabla 5). Este resultado podría ser atribuido tanto a factores temporales como geográficos, dada la distancia (ca. $38 \mathrm{~km}$ ) entre el sitio Toconao 
Rocío lópez-Barrales, Mark Hubbe, Eugenio Aspillaga, Walter A. Neves, Hermann M. Niemeyer

\begin{tabular}{|c|c|c|c|c|c|}
\hline \multicolumn{6}{|l|}{ a. Cervical } \\
\hline & SC & Grados de libertad & $\mathrm{MC}$ & $\mathrm{F}$ & $\mathrm{P}$ \\
\hline Sitio & 6,278 & 3 & 2,093 & 6,125 & $<0,001$ \\
\hline Sexo & 0,051 & 1 & 0,051 & 0,149 & 0,700 \\
\hline Edad & 6,576 & 1 & 6,576 & 19,247 & $<0,0 \circ 1$ \\
\hline Sitio*Sexo & 2,045 & 3 & 0,682 & 1,995 & 0,118 \\
\hline Sitio*Edad & 4,315 & 3 & 1,438 & 4,210 & 0,007 \\
\hline Error & 42,367 & 124 & 0,342 & & \\
\hline \multicolumn{6}{|c|}{ b. Torácico ( $\left.\mathrm{T}_{1}-\mathrm{T} 6\right)$} \\
\hline & SC & Grados de libertad & $\mathrm{MC}$ & $\mathrm{F}$ & $\mathrm{P}$ \\
\hline Sitio & 3,066 & 3 & 1,022 & 4,909 & 0,003 \\
\hline Sexo & 0,252 & 1 & 0,252 & 1,210 & 0,274 \\
\hline Edad & 2,581 & 1 & 2,581 & 12,394 & $<0,001$ \\
\hline Sitio*Sexo & 0,395 & 3 & 0,132 & 0,632 & 0,596 \\
\hline Sitio*Edad & 2,456 & 3 & 0,819 & 3,932 & 0,011 \\
\hline Error & 20,198 & 97 & 0,208 & & \\
\hline \multicolumn{6}{|c|}{ c. Torácico $\left(\mathrm{T}_{7}-\mathrm{T}_{12}\right)$} \\
\hline & $\mathrm{SC}$ & Grados de libertad & MC & $\mathrm{F}$ & $\mathrm{P}$ \\
\hline Sitio & 4,602 & 3 & 1,534 & 3,914 & 0,010 \\
\hline Sexo & 0,393 & 1 & 0,393 & 1,003 & 0,319 \\
\hline Edad & 8,585 & 1 & 8,585 & 21,903 & $<0,001$ \\
\hline Sitio*Sexo & 1,781 & 3 & 0,594 & 1,515 & 0,214 \\
\hline Sitio*Edad & 1,574 & 3 & 0,525 & 1,338 & 0,265 \\
\hline Error & 47,035 & 120 & 0,392 & & \\
\hline \multicolumn{6}{|l|}{ d. Lumbar } \\
\hline & SC & Grados de libertad & MC & $\mathrm{F}$ & $\mathrm{P}$ \\
\hline Sitio & 4,522 & 3 & 1,507 & 2,698 & 0,049 \\
\hline Sexo & 0,00086 & 1 & 0,00086 & 0,0015 & 0,969 \\
\hline Edad & 18,767 & 1 & 18,767 & 33,594 & $<0,0 \circ 1$ \\
\hline Sitio*Sexo & 2,701 & 3 & 0,900 & 1,612 & 0,190 \\
\hline Sitio*Edad & 4,573 & 3 & 1,524 & 2,729 & 0,047 \\
\hline Error & 68,154 & 122 & 0,559 & & \\
\hline
\end{tabular}

Tabla 4. Resumen de las pruebas de significancia dentro del modelo lineal generalizado para cada segmento vertebral. Los valores de P menores a 0,05 se destacan en negrita. 

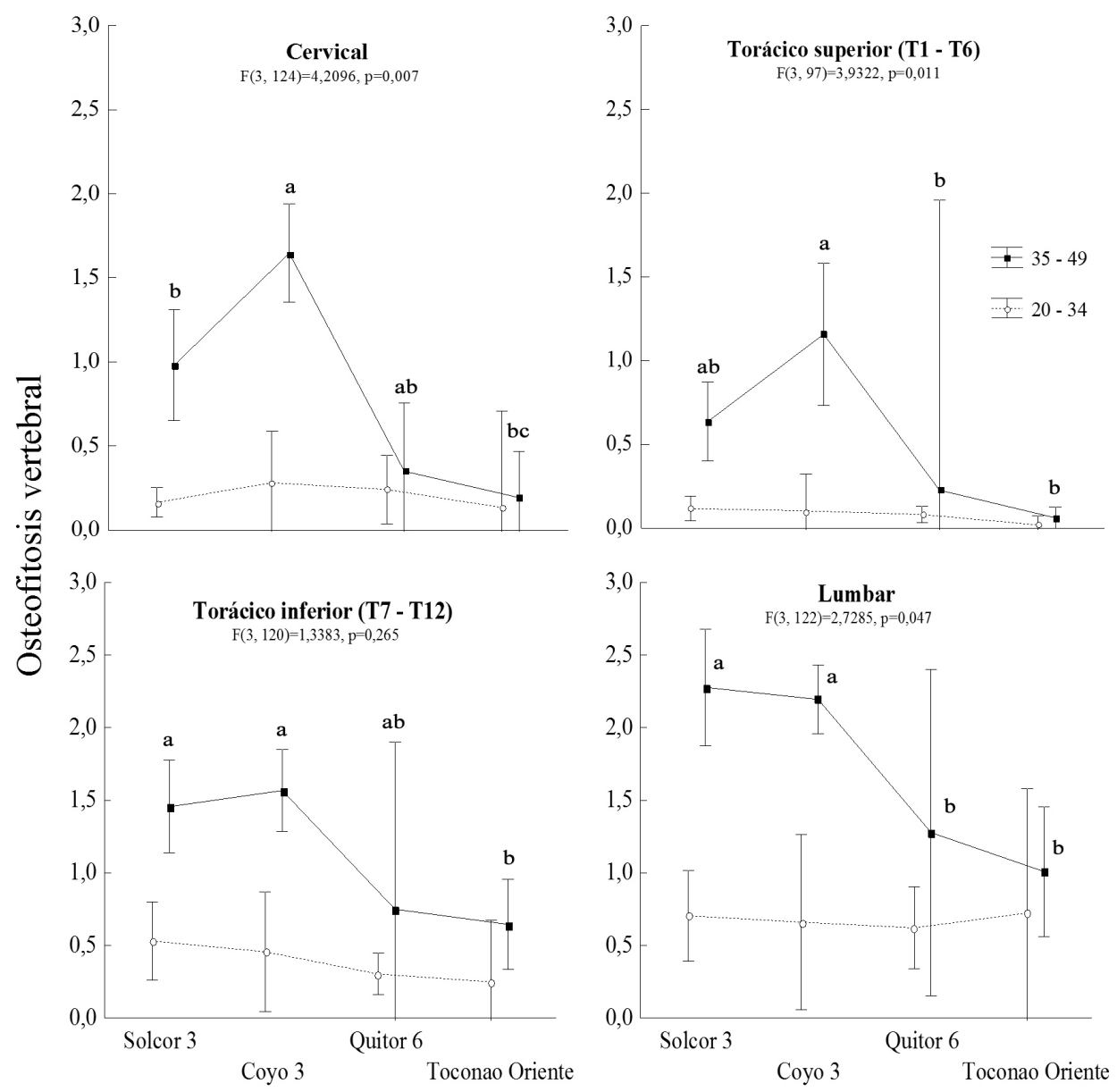

\section{Sitios}

Figura 4. Interacciones entre edad y sitio en grado de osteofitosis (promedios e intervalos de confianza de 0,95) de cada segmento vertebral. Los puntos de las dos series de edad para cada sitio están levemente desplazados en el eje de las abscisas para facilitar la interpretación. Los conjuntos de letras sobre los puntos correspondientes al rango etario más viejo representan las comparaciones estadísticas entre los sitios; si los conjuntos de letras tienen elementos en común, los resultados para esos sitios no son significativamente diferentes.

Oriente y el pueblo actual de San Pedro de Atacama; sin embargo, ya que Toconao Oriente no se diferenció significativamente de Quitor 6 (Figura 4 y Tabla 5), ubicado en los oasis centrales, las diferencias probablemente se deban a factores temporales $y / o$ culturales.

Las investigaciones arqueológicas han planteado al período Medio como un momento cuando la influencia Tiwanaku estuvo acompañada de un incremento en el desarrollo cultural local con una mayor riqueza material y participación activa en una gran red de tráfico interregional (Berenguer y Dauelsberg 1989; Núñez 2007). Algunos estudios han mostrado que esta prosperidad tuvo implicancias en la calidad de vida de las poblaciones atacameñas, planteando mejoras durante el período Medio (Neves y Costa 1998; Costa et al. 2004), aunque otros indicadores han mostrado un panorama distinto (Costa et al. 1998; Tótora da Glória et al. 2011).

Los datos entregados sobre osteofitosis vertebral confirman la complejidad del problema, ya que por un lado muestran una discordancia con los estudios de traumas postcraneales (Costa et al. 1998, 2004), y por otro, apoyan las diferencias detectadas en robusticidad (Pomeroy 2013). Según Costa et al. $(1998,2004)$ las actividades cotidianas y los riesgos asociados a ellas no cambiaron 
Rocío lópez-Barrales, Mark Hubbe, Eugenio Aspillaga, Walter A. Neves, Hermann M. Niemeyer

\begin{tabular}{|c|c|c|c|c|}
\hline \multicolumn{5}{|c|}{ Segmento cervical } \\
\hline \multicolumn{5}{|c|}{ Error entre EMC $=0,54 ;$ g.l. $=66$} \\
\hline Sitio & Solcor 3 & Coyo 3 & Quitor 6 & $\begin{array}{l}\text { Toconao } \\
\text { Oriente }\end{array}$ \\
\hline $\begin{array}{l}\text { Solcor } 3 \\
(0,980)\end{array}$ & $\mathrm{X}$ & & & \\
\hline $\begin{array}{l}\text { Coyo } 3 \\
(1,647)\end{array}$ & 0,008 & $\mathrm{x}$ & & \\
\hline $\begin{array}{l}\text { Quitor } 6 \\
(0,350)\end{array}$ & 0,626 & 0,072 & $\mathrm{x}$ & \\
\hline $\begin{array}{l}\text { Toconao } \\
\text { Oriente } \\
(0,192)\end{array}$ & 0,091 & 0,000 & 0,990 & $\mathrm{X}$ \\
\hline \multicolumn{5}{|c|}{ Segmento torácico superior $\left(\mathrm{T}_{1}-\mathrm{T} 6\right)$} \\
\hline \multicolumn{5}{|c|}{ Error entre EMC $=0,39 ;$ g.l $=50$} \\
\hline Sitio & Solcor 3 & Coyo 3 & Quitor 6 & $\begin{array}{l}\text { Toconao } \\
\text { Oriente }\end{array}$ \\
\hline $\begin{array}{l}\text { Solcor } 3 \\
(0,637)\end{array}$ & $\mathrm{X}$ & & & \\
\hline $\begin{array}{l}\text { Coyo } 3 \\
(1,158)\end{array}$ & 0,073 & X & & \\
\hline $\begin{array}{c}\text { Quitor } 6 \\
(0,227)\end{array}$ & 0,914 & 0,454 & $\mathrm{x}$ & \\
\hline $\begin{array}{l}\text { Toconao } \\
\text { Oriente } \\
(0,062)\end{array}$ & 0,184 & 0,002 & 0,994 & $\mathrm{X}$ \\
\hline
\end{tabular}

\begin{tabular}{|c|c|c|c|c|}
\hline \multicolumn{5}{|c|}{ Segmento torácico inferior $\left(\mathrm{T}_{7}-\mathrm{T}_{12}\right)$} \\
\hline \multicolumn{5}{|c|}{ Error entre $\mathrm{EMC}=0,50$; g.l. $=63$} \\
\hline Sitio & Solcor 3 & Coyo 3 & Quitor 6 & $\begin{array}{l}\text { Toconao } \\
\text { Oriente }\end{array}$ \\
\hline $\begin{array}{c}\text { Solcor } 3 \\
(1,456)\end{array}$ & $\mathrm{x}$ & & & \\
\hline $\begin{array}{l}\text { Coyo } 3 \\
(1,567)\end{array}$ & 0,947 & $\mathrm{x}$ & & \\
\hline $\begin{array}{l}\text { Quitor 6 } \\
(0,750)\end{array}$ & 0,622 & 0,503 & $\mathrm{x}$ & \\
\hline $\begin{array}{l}\text { Toconao } \\
\text { Oriente } \\
(0,646)\end{array}$ & 0,048 & 0,019 & 0,998 & $\mathrm{X}$ \\
\hline \multicolumn{5}{|c|}{ Segmento lumbar } \\
\hline \multicolumn{5}{|c|}{ Error entre EMC $=0.58 ;$ g.l. $=66$} \\
\hline Sitio & Solcor 3 & Coyo 3 & Quitor 6 & $\begin{array}{l}\text { Toconao } \\
\text { Oriente }\end{array}$ \\
\hline $\begin{array}{l}\text { Solcor } 3 \\
(2,277)\end{array}$ & $\mathrm{X}$ & & & \\
\hline $\begin{array}{l}\text { Coyo } 3 \\
(2,194)\end{array}$ & 0,982 & X & & \\
\hline $\begin{array}{c}\text { Quitor 6 } \\
(1,277)\end{array}$ & 0,388 & 0,465 & $\mathrm{X}$ & \\
\hline $\begin{array}{l}\text { Toconao } \\
\text { Oriente } \\
(1, \infty \circ 9)\end{array}$ & 0,001 & 0,002 & 0,973 & X \\
\hline
\end{tabular}

Tabla 5. Resultados de las comparaciones a posteriori en el rango etario 35 - 49 años: prueba de comparación de medias en grupos desiguales ("unequal N HSD") (significación a P<0,05). Los valores entre paréntesis corresponden al grado de osteofitosis vertebral promedio de cada sitio para el rango etario 35 - 49 años. Los valores de P menores a 0,05 se destacan en negrita.

durante todo el desarrollo cultural. Sin embargo, la osteofitosis vertebral indica un mayor estrés físico sobre la espalda durante el período Medio, planteando así un uso más intenso del cuerpo en actividades relacionadas con la carga sobre la columna. Esto resulta particularmente interesante, pues indica que solo algunos de los aspectos relacionados con la calidad y estilo de vida se beneficiaron con la influencia del estado altiplánico y del momento próspero que vivían las poblaciones locales.

Algunos estudios han planteado que la osteofitosis vertebral estaría relacionada con la carga mecánica dada por el peso corporal en conjunto con las actividades físicas (Kellgren y Lawrence 1952; Bridges 1994; O’Neill et al.
1999; Sofaer Derevenski 2000; Schmitt et al. 2004). Los resultados presentados sugerirían entonces que durante el período Intermedio Tardío las poblaciones sufrieron un estrés biomecánico en la espalda menor con respecto a las poblaciones que vivieron durante el período Medio, producto de un cambio posiblemente en el tipo o en la intensidad de las actividades físicas desarrolladas.

Las poblaciones atacameñas, desde su establecimiento como poblados permanentes hasta la llegada de los conquistadores españoles, se dedicaron principalmente a la agricultura, el pastoreo y el intercambio mediante el caravaneo (Núñez 2007). No se han detectado cambios significativos en el tipo de actividades durante todo el 
desarrollo agroalfarero, por lo que probablemente las diferencias en el grado de osteofitosis vertebral se deban a cambios en la intensidad que se le dio a estas actividades a través del tiempo. Los cambios de intensidad pueden ser atribuidos a cambios en aspectos socioeconómicos que provocaron una mayor o menor demanda de trabajo o a cambios tecnológicos que afectaron la energía invertida en cada una de estas actividades y/o la forma de emplearla, como por ejemplo, cambios en el trabajo agrícola por la llegada de alguna nueva tecnología o manera de cultivo o cambios en la forma de transporte de carga que disminuyeron la carga de trabajo.

Una actividad que se ha relacionado con la osteofitosis vertebral son las formas de transporte de carga sobre la espalda (Bridges 1994; Sofaer Derevenski 2000). La intensidad de osteofitosis detectada en San Pedro de Atacama podría entonces explicarse por cambios en la intensidad o en los métodos de la actividad de carga y transporte entre el período Medio e Intermedio Tardío. El hallazgo de capachos (tipo de bolso que se lleva en la espalda y se sostiene por una correa que se apoya en la frente), es común en contextos arqueológicos del área siendo utilizados hasta el día de hoy en algunas áreas de los Andes (Gerszten et al. 2001; Briones et al. 2005; Cases et al. 2008). Los resultados presentados podrían estar relacionados con el transporte de carga sobre la cabeza o utilizando ésta como soporte.

La intensidad de la osteofitosis en el segmento cervical en Coyo 3 es más alta que en Solcor 3. Como fue mencionado anteriormente, Coyo 3 se corresponde con el momento en que empiezan a decaer las relaciones con Tiwanaku, lo que pudo traer desajustes socioeconómicos y por lo tanto cambios en las actividades desarrolladas por las poblaciones. La diferencia entre Coyo 3 y Solcor 3 solo se observa en la columna cervical por lo que si hubo un cambio en las actividades, éste estaría relacionado con el uso o a la carga sobre este segmento vertebral. El hecho que San Pedro dejara de ser parte de una red de tráfico a larga distancia manejada por Tiwanaku pudo provocar, especialmente, un aumento en los desplazamientos o una mayor demanda de trabajo. Esta explicación también es apoyada por la mayor robusticidad de los miembros inferiores detectada durante la transición entre el período Medio y el Intermedio Tardío con respecto al período Medio (Pomeroy 2013).
La intensidad de osteofitosis vertebral observada en Coyo 3 también podría estar relacionada con actividades específicas de algunos oasis. Algunas investigaciones arqueológicas han notado la presencia de instrumentos relacionados con la minería, especialmente en el oasis de Coyo (Costa y Llagostera 1994; Núñez 1999; Salazar et al. 2011; Figueroa et al. 2013). Arriaza (1991) analizó los restos de Coyo Oriente y sugirió que las condiciones osteoartríticas observadas en la articulación de la rodilla podrían estar relacionadas con el trabajo en minería. Por otra parte, una investigación en una población contemporánea mostró que los trabajadores de una mina de carbón presentaban niveles mayores de degeneración del disco intervertebral (incluyendo osteofitos), que trabajadores de otras actividades manuales y de oficina (Kellgren y Lawrence 1952), lo que sugiere que las actividades mineras provocan una mayor carga mecánica sobre la espalda. Si bien las herramientas encontradas en Coyo 3 no muestran señales de uso y son menos frecuentes que en otros sitios del mismo sector (Costa y Llagostera 1994), los resultados bioarqueológicos de Arriaza (1991) y los estudios arqueológicos sobre minería prehispánica (Salazar et al. 2011; Figueroa et al. 2013) podrían explicar las diferencias detectadas en Coyo 3, ya que actividades específicas como la minería pudieron haber traído como consecuencia un mayor desarrollo de condiciones osteoartríticas. Lamentablemente, existe una escasa contraparte de datos arqueológicos directos que avalen o rechacen lo planteado; por lo mismo, es importante que se desarrollen estudios arqueológicos en torno a este tema que busquen posibles cambios que puedan incidir sobre la prevalencia de la osteofitosis vertebral. Así mismo, el análisis de otras articulaciones de los mismos individuos analizados en este estudio, podrían confirmar o rechazar lo planteado, ya que un enfoque más informativo se consigue utilizando todo el esqueleto para registrar los patrones de osteoartritis y osteofitosis vertebral (Jurmain 1990; Goodman y Martin 2002).

Estudios previos en restos esqueletales han mostrado diferencias entre hombres y mujeres en lesiones y cambios degenerativos en la columna vertebral (Bridges 1989; Sofaer Derevenski 2000; Klaus et al. 2009; Woo y Sciulli 2013). Estas diferencias han permitido plantear una división del trabajo relacionada con el género. Los resultados de nuestro estudio sin embargo no muestran que el sexo sea un factor significativo en los cambios degenerativos 
registrados en los cuerpos vertebrales de las muestras de San Pedro de Atacama de ningún período. Es interesante que en una sociedad compleja como la atacameña, durante y después del período Medio, no se observen diferencias asociadas al sexo en una variable que refleja actividad física, como la osteofitosis.

Otros indicadores de estilo de vida en San Pedro de Atacama como los traumas postcraneales tampoco muestran diferencias relacionadas con el sexo de los individuos, lo cual indicaría que los riesgos asociados a las actividades cotidianas fueron similares para toda la población atacameña (Costa et al. 1998). Lo mismo sucede con la osteoartritis de rodilla vista por Arriaza en el sitio Coyo Oriente, donde la similitud de este indicador en hombres y mujeres sugiere que todas las personas de ese sitio participaban en actividades relacionadas con la minería (Arriaza 1991). Sin embargo, Pomeroy (2013) encontró diferencias significativas en la robusticidad y la sección transversal de los huesos de las extremidades inferiores entre hombres y mujeres de San Pedro de Atacama durante el período Medio, y lo interpreta como un nivel mayor de movilidad de los hombres posiblemente en actividades relacionadas con el intercambio a larga distancia y/o pastoreo. Al parecer, la sociedad atacameña no hacía distinciones entre sexos en una amplia variedad de actividades físicas cotidianas durante los períodos estudiados, aunque sí lo hacía en otras actividades posiblemente relacionadas con el intercambio a larga distancia durante el período de mayor influencia de Tiwanaku (Pomeroy 2013) y en otros aspectos de la vida tales como estatus o dieta (Hubbe et al. 2012).

La influencia de Tiwanaku durante el período Medio no fue homogénea en toda la población atacameña, observándose diferencias sociales tanto en la distribución de las ofrendas funerarias como en los patrones de salud y violencia (Torres-Rouff 2011; Hubbe et al. 2012). TorresRouff (2011) sugiere que se generaron ciertas tensiones sociales al no beneficiarse toda la población atacameña con esta influencia. Los datos de osteofitosis vertebral no muestran diferencias significativas entre dos grupos con mayor o menor estatus social (Solcor 3 élite y Solcor 3 no élite, respectivamente). Esto podría ser interpretado como que las diferencias sociales presentes en este período no se relacionaron con las actividades físicas realizadas por estos grupos. De este modo, se puede plantear que la presencia de Tiwanaku en la zona no influyó sobre la diferenciación de roles en las actividades cotidianas y, por extensión, productivas, dentro de los grupos atacameños, de modo que los individuos de estatus social más alto estarían realizando más o menos las mismas actividades que el resto de los individuos. Al parecer, Tiwanaku contribuyó a segmentar la población, pero no a cambiarle su estilo de vida, ya que toda la población realizaría actividades similares.

Investigaciones en torno a la degeneración del disco intervertebral plantean que el factor genético es importante en esta condición (Kalichman 2010). Si se entiende la aparición y severidad de los osteofitos vertebrales como una característica de este proceso degenerativo, las diferencias encontradas en la muestra de San Pedro de Atacama podrían ser explicadas por diferencias genéticas entre los grupos humanos que habitaron los oasis en estos dos períodos, por ejemplo, por causa del aporte poblacional desde otras regiones con las cuales mantenían contactos. Aunque algunos estudios muestran diferencias en diversidad fenotípica entre las distintas fases culturales de la prehistoria de San Pedro de Atacama que podrían ser consecuencia de migraciones desde otras zonas (Cocilovo 1981; Cocilovo y Rothhammer 1990; Varela y Cocilovo 2000, 2009), otros estudios referidos a deformación craneana e isótopos estables han encontrado evidencia de la presencia en San Pedro de Atacama de solo escasos individuos de origen foráneo (Knudson 2007; Varela y Cocilovo 2007; Knudson y Torres-Rouff 2009; Torres-Rouff et al. 2013). En resumen, no existen datos que indiquen cambios significativos en la composición genética de la población, más allá de un aumento de la diversidad presente, que puedan explicar las diferencias encontradas en osteofitosis.

Un punto importante a considerar en todo estudio sobre muestras esqueletales del pasado es la Paradoja Osteológica (Wood et al. 1992). Aunque la Paradoja Osteológica presenta limitaciones importantes para la interpretación de la prevalencias de paleopatologías, creemos que para el caso de la osteofitosis en San Pedro de Atacama, nuestras interpretaciones de los datos no están seriamente afectadas por los problemas levantados por Wood et al. (1992).

En primer lugar, la osteofitosis vertebral, al ser una enfermedad degenerativa progresiva, no se relaciona directamente con la mortalidad y, por lo mismo, no está 
asociada con tasas diferenciales de mortalidad. En otras palabras, no existe la posibilidad de que individuos mueran debido a la osteofitosis, antes que la osteofitosis se refleje en su esqueleto.

Ahora bien, como la osteofitosis vertebral es degenerativa los perfiles de edad podrían efectivamente afectar los resultados observados. Sin embargo, el enfoque estadístico elegido permite salvar este obstáculo pues expone la influencia de los distintos factores de manera independiente, corrigiendo el efecto de la edad en las comparaciones entre sitios, destacando claramente la interacción que existe entre edad y sitio; es decir, las diferencias entre prevalencia de osteofitosis entre sitios solo se manifiestan en adultos mayores (35 - 49 años).

Finalmente, un tema que subyace a gran parte de los estudios sobre poblaciones del pasado es la representatividad de las muestras. En el caso de este estudio se evidencia el desequilibrio de las series esqueletales utilizadas, donde las muestras del período Intermedio Tardío son menos numerosas que las del período Medio, y una de ellas, Quitor 6, además presenta particular heterogeneidad en el tamaño de los grupos etarios. Sin embargo, la inclusión de otra serie esqueletal de este período (Toconao Oriente), la ausencia de diferencias entre sexos, y el enfoque estadístico elegido permitieron superar en parte este problema, mostrando que existen patrones importantes de diferencias entre sitios del período Medio y del Intermedio Tardío.

\section{$*$ Conclusión}

En resumen, los resultados obtenidos en esta investigación comprueban el carácter degenerativo de la osteofitosis vertebral, ya que la edad influye directamente en las diferencias observadas entre los distintos sitios. Sin embargo, aun controlando el factor etario es posible observar diferencias significativas entre los distintos sitios analizados, que se muestran evidentes entre adultos mayores (35 - 49 años), revelando posibles diferencias en los patrones de actividad a lo largo del tiempo. Estas diferencias podrían ser explicadas por cambios en la intensidad de la carga mecánica ejercida en la columna vertebral, ya sea por distintos tipos de actividades o por cambios en la intensidad de las actividades desarrolladas por las poblaciones de San Pedro de Atacama, como podrían ser las actividades relacionadas con una carga axial sobre el cuerpo (uso de capacho o carga sobre la espalda).

Un caso particular sucede con el sitio Coyo 3, especialmente en su segmento cervical. Sus niveles de osteofitosis vertebral son altos respecto a los otros sitios, lo que podría relacionarse con dos aspectos de carácter cultural. Por un lado, este sitio se ubicaría cronológicamente en el momento en que se produce un desajuste social y económico por la desarticulación de la hegemonía de Tiwanaku. Por otro lado, el hallazgo de herramientas para el trabajo de minería en los sitios de Coyo, podría estar indicando una especialización en actividades mineras (las que incluyen carga de objetos pesados). Esta última interpretación queda solo como una hipótesis ya que los datos actualmente son insuficientes para llegar a conclusiones.

Un enfoque que puede entregar mayor información es la investigación sistemática de patrones de osteoartritis en distintas articulaciones, usando toda la muestra esqueletal (Jurmain 1990; Goodman y Martin 2002). Por lo mismo y para llegar a conclusiones más robustas sería necesario realizar en el futuro un análisis de las articulaciones apendiculares de las mismas muestras utilizadas en este estudio.

Agradecimientos Esta investigación fue financiada por el programa CONICYT-PIA, proyecto Anillo en Ciencia y Tecnología ACT Nº 09 titulado "Interacciones y movilidad humanas en poblaciones prehispánicas del norte y centro de Chile: un enfoque integrador para las Ciencias Sociales utilizando marcadores biomédicos, genéticos, químicos y mineralógicos". Agradecemos a los evaluadores anónimos por sus constructivas sugerencias que permitieron mejorar este trabajo. 


\section{$*$ Referencias Citadas}

ADAMS, M. A. 2012. Basic science of spinal degeneration. Surgery (Oxford) 30:347-350.

ADAMS, M. A., P. DOLAN y D. S. MCNALLY. 2009. The internal mechanical functioning of intervertebral discs and articular cartilage, and its relevance to matrix biology. Matrix Biology 28:384-389.

ARRIAZA, B. 1991. Paleo-salud de los habitantes de Coyo Oriental: San Pedro de Atacama. Interpretive Research Project RO 21963-89. Washington D. C. (Documento inédito).

BATTIÉ, M. C., T. VIDEMAN, J. KAPRIO, L. E. GIBBONS, K. GILL, H. MANNINEN, J. SAARELA, y L. PELTONEN. 2009. The Twin Spine Study: contributions to a changing view of disc degeneration. Spine Journal 9: 47-59.

BERENGUER, J. y P. DAUELSBERG. 1989. El Norte Grande en la órbita de Tiwanaku (400 a 1.200 d. C.). En Prehistoria: desde sus orígenes hasta los albores de la conquista, J. Hidalgo, V. Schiappacasse, H. Niemeyer, C. Aldunate e I. Solimano (Eds.), pp. 129-180. Editorial Andrés Bello, Santiago de Chile.

BERENGUER, J., A. ROMAN, A. DEZA y A. LlAGOSTERA. 1988. Testing a cultural sequence for the Atacama Desert. Current Anthropology 29: 341-346.

BRAVO, L. y A. LLAGOSTERA. 1986. Solcor-3: Un aporte al conocimiento de la cultura San Pedro. Período 500 al 900 d.C. Chungara 16-17: 323-332.

BRIDGES, P. S. 1989. Spondylolysis and its relationship to degenerative joint disease in the prehistoric Southeastern United States. American Journal of Physical Anthropology 79:321-329.

1992. Prehistoric arthritis in the Americas. Annual Review of Anthropology 21: 67-91.

1994. Vertebral arthritis and physical activities in the prehistoric Southeastern United States. American Journal of Physical Anthropology 93: 83-93.

BRIONES, L., L. NÚÑEZ y V. G. STANDEN. 2005. Geoglifos y tráfico prehispánico de caravanas de llamas en el desierto de Atacama (norte de Chile). Chungara. Revista de Antropología Chilena 37: 195-223.

CASES, B., C. REES, G. PIMENTEL, R. LABARCA y D. LEIVA. 2008. Sugerencias desde un contexto funerario en un espacio vacío del desierto de Atacama. Boletín del Museo Chileno de Arte Precolombino 13: 51-70.
COCILOVO, J. A. 1981. Estudio sobre discriminación y clasificación de poblaciones pre-hispánicas del N.O. Argentino. Museo Nacional de Historia Natural, Santiago de Chile.

COCILOVO, J. A. y F. ROTHHAMMER. 1990. Paleopopulation biology of the southern Andes: craniofacial chronological and geographical differentiation. Homo 41: 16-31.

COSTA, M. A. 1988. Reconstitución física y cultural de la población tardía del cementerio Quitor 6 (San Pedro de Atacama). Estudios Atacameños 9: 107-135.

COSTA, M. A. y A. LlagosterA. 1994. Coyo-3: momentos finales del período medio en San Pedro de Atacama. Estudios Atacameños 11: 73.107.

COSTA, M. A., W. A. NEVES y M. HUBBE. 2004. Influencia de Tiwanaku en la calidad de vida biológica de la población prehistórica de San Pedro de Atacama. Estudios Atacameños 27: $103-116$.

COSTA, M. A., W. A. NEVES, A. M. DE BARROS y R. BARTOLOMUCCI. 1998. Trauma y estrés en poblaciones prehistóricas de San Pedro de Atacama, Norte de Chile. Chungará 30: 65-74.

FIGUEROA, V., D. SALAZAR, H. SALINAS, P. NÚÑEZ-REGUEIRO y G. MANRÍQUEZ. 2013. Pre-hispanic mining ergology of northern Chile: an archaeological perspective. Chungara. Revista de Antropología Chilena 45 (1): 61-81.

GERSZTEN, P. C., E. E. GERSZTEN y M. J. ALLISON. 2001. Diseases of the spine in South American mummies. Neurosurgery 48 (1): 208-213.

GOODMAN, A. H. y D. L. MARTIN. 2002. Reconstructing health profiles from skeletal remains. En The Backbone of History: Health and Nutrition in the Western Hemisphere, R. Steckel y J. Rose (Eds.). Cambridge University Press, Cambridge, New York.

HAIR, J. F., W. C. BLACK, B. J. BABIN y R. E. ANDERSON. 2009. Multivariate Data Analysis, 7th edition. Pearson Education, Upper Saddle River, NJ.

HUBBE, M., C. TORRES-ROUFF, W. A. NEVES, L. M. KING, P. J. TÓTORA DA GLÓRIA y M. A. COSTA. 2012. Dental health in northern Chile's Atacama oases: evaluating the Middle Horizon (AD 500-1000) impact on local diet. American Journal of Physical Anthropology 148 (1): 62-72. 
JURMAIN, R. D. 1990. Paleoepidemiology of a central California prehistoric population from CA-Ala-329. II. Degenerative disease. American Journal of Physical Anthropology 83: 83-94.

JURMAIN, R. D. y L. KILGORE. 1995. Skeletal evidence of osteoarthritis: a palaeopathological perspective. Annals of the Rheumatic Diseases 54: 443-450.

KALICHMAN, L. 2010. The Etiology of Intervertebral Disc Degeneration. IBMS BoneKEy 7: 388-405.

KELLGREN, J. H. y J. S. LAWRENCE. 1952. Rheumatism in miners. Part II: X-ray Study. British Journal of Indian Medicine 9: 197-207.

1955. Rheumatism in coal miners: part III: Occupational factors. British Journal of Indian Medicine 12: 249-261.

KELLGREN, J. H.y J. S. LAWRENCE. 1958. Osteo-arthrosis and disc degeneration in an urban population. Annals of Rheumatic Diseases 17: 388-397.

KENNEDY, K. A.R. 1989. Skeletal markers of occupational stress. En Reconstruction of life from the skeleton, M.Y. Ican y K. Kennedy (Eds.), pp. 129-160. Liss, Nueva York.

KIM, D. K., M. J. KIM, Y. S. KIM, C. S. OH y D. H. SHIN. 2012. Vertebral osteophyte of pre-modern Korean skeletons from Joseon tombs. Anatomy \& Cell Biology 45 (4): 274-281.

KLAUS H.D., C.S. LARSEN y M.E. TAM. 2009. Economic intensification and degenerative joint disease: life and labor on the postcontact north coast of Peru. American Journal of Physical Anthropology 139: 204-221.

KNUDSON, K. 2007. La influencia de Tiwanaku en San Pedro de Atacama: Una investigación utilizando el análisis de isótopos del estroncio. Estudios Atacameños 33: 7-24.

KNUDSON, K. y C. TORRES-ROUFF, 2009. Investigating cultural heterogeneity and multiethnicity in San Pedro de Atacama, Northern Chile, through biogeochemistry and bioarchaeology. American Journal of Physical Anthropology 138: 473-485.

KNÜSEL, C. J., S. GÖGGEL y D. LUCY. 1997. Comparative Degenerative Joint Disease of the Vertebral Column in the Medieval Monastic Cemetery of the Gilbertine Priory of St. Andrew, Fishergate, York, England. American Journal of Physical Anthropo$\log$ 103: 481-495.

KUMARESAN, S., N. YOGANANDAN, F. A. PINTAR, D. J. MAIMAN y V. K. GOEL. 2001. Contribution of disc degeneration to osteophyte formation in the cervical spine: a biomechanical investigation. Journal of Orthopaedic Research 19: 977-984.
LE PAIGE, G. 1965. San Pedro de Atacama y su Zona. Anales de la Universidad del Norte 4.

LESSA, A. y S. MENDONÇA DE SOUZA. 2003. Paleoepidemiologia dos traumatismos cotidianos em Solcor-3, San Pedro de Atacama, Chile: Riscos diferenciados no período Tiwanaku. Antropologia Portuguesa 20 (21): 183-206.

LESSA, A. y S. MENDONÇA DE SOUZA. 2004. Violence in the Atacama Desert during the Tiwanaku Period: Social Tension? International Journal of Osteoarchaeology 14: 374-388.

LlagosterA, A., M. C. TORRES y M. A. COSTA. 1988. El complejo psicotrópico en Solcor 3 (San Pedro de Atacama). Estudios Atacameños 9: 67-106.

NATHAN, H. 1962. Osteophytes of the vertebral column. The Journal of Bone and Joint Surgery 44: 243-268.

NEVES, W. A. y M. A. COSTA. 1998. Adult stature and standard of living in prehistoric Atacama Desert, Northern Chile. $\mathrm{Cu}$ rrent Anthropology 39: 278-281.

NÚÑEZ, L. 1999. Valoración minero-metalúrgica circumpuneña: menas y mineros para el Inka rey. Estudios Atacameños 18: 177222.

2007. Vida y cultura en el oasis de San Pedro de Atacama. Editorial Universitaria, Santiago de Chile.

OISHI, Y., K. SHIMIZU, T. KATOH, H. NAKAO, M. YAMAURA, T. FURUKO, K. NARUSAWA y T. NAKAMURA. 2003. Lack of association between lumbar disc degeneration and osteophyte formation in elderly Japanese women with back pain. Bone 32: 405-411.

O'NEILL, T. W., E. V. MCCLOSKEY, J. A. KANIS, A. K. BHALLA, J. REEVE, D. M. REID y A. J. SILMAN. 1999. The distribution, determinants, and clinical correlates of vertebral osteophytosis: a population based survey. Journal of Rheumatology 26: 842-848.

ORTNER, D. J. 2003. Identification of Pathological Conditions in Human Skeletal Remains. Academic Press, San Diego.

POMEROY, E. 2013. Biomechanical insights into activity and long distance trade in the south-central Andes (AD 5001450). Journal of Archaeological Science 40 (8): 3129-3140.

ROJAS-SEPÚLVEDA, C., Y. ARDAGNA y O. DUTOUR. 2008. Paleoepidemiology of Vertebral Degenerative Disease in a PreColumbian Muisca Series from Colombia. American Journal of Physical Anthropology 135: 416-430. 
SAlAZAR, D., V. FIGUEROA, D. MORATA, B. Mille, G. MANRÍQUEZ y A. CIFUENTES. 2011. Metalurgia en San Pedro de Atacama Durante el Período Medio: Nuevos Datos, Nuevas Preguntas. Revista de Antropología 23: 123-148.

SCHIAPPACASSE, V., V. CASTRO y H. NIEMEYER. 1989. Los desarrollos regionales en el Norte Grande. En Culturas de Chile. Prehistoria, J. Hidalgo, V. Schiappacasse, H. Niemeyer, C. Aldunate e I. Solimano (Eds.), pp. 181-220. Editorial Andrés Bello, Santiago de Chile.

SCHMITT, H., E. DUBLJANIN, S. SCHNEIDER y M. SCHILTENWOLF. 2004. Radiographic changes in the lumbar spine in former elite athletes. Spine 29: 2554-2559.

SOFAER DEREVENSKI, J. R. 2000. Sex differences in activityrelated osseous change in the spine and the gendered division of labor at Ensay and Wharram Percy, UK. American Journal of Physical Anthropology 111: 333-354.

TORRES-ROUFF, C. 2008. The influence of Tiwanaku on life in the Chilean Atacama: mortuary and bodily perspectives. American Anthropologist 110: 325-337.

2011. Hiding Inequality Beneath Prosperity: Patterns of Cranial Injury in Middle Period San Pedro de Atacama, Northern Chile. American Journal of Physical Anthropology 146: 28-37.

TORRES-ROUFF, C. y M. A. COSTA. 2006. Interpersonal violence in prehistoric San Pedro de Atacama, Chile: Behavioral implications of environmental stress. American Journal of Physical Anthropology 130: 60-70.

TORRES-ROUFF, C. y M. HUBBE. 2013. The sequence of human occupation in the Atacama oases, Chile: A radiocarbon chronology based on human skeletal remains. Latin American Antiquity 24:330-344.

TORRES-ROUFF, C., K. KNUDSON y M. HUBBE. 2013. Issues of affinity: Exploring population structure in the middle and regional developments periods of San Pedro de Atacama, Chile. American Journal of Physical Anthropology 152 (3):370-382.

TÓTORA DA GLÓRIA, P. J., W. A. NEVES, M. A. COSTAy R. BARTOLOMUCCI. 2011. Enfermedades infecciosas no-específicas en poblaciones prehistóricas de San Pedro de Atacama, Norte de Chile. Chungara. Revista de Antropología Chilena 43: 135-146.

UBELAKER, D. H. y BUIKSTRA, J. E. 1994. Standards for data collection from human skeletal remains. Arkansas Archeological Survey Research, Series 44.

URIBE, M. 2002. Sobre alfarería, cementerios, fases y procesos durante la prehistoria tardía del desierto de Atacama (8001600 DC). Estudios Atacameños 22: 7-31.

URIBE, M., L. ADAN y C. AGÜERO, 2004. Arqueología de los períodos Intermedio Tardío y Tardío de San Pedro de Atacama y su relación con la cuenca del río Loa. Chungara. Revista de Antropología Chilena 36, especial 2: 943-956.

VAN DER MERWE, A. E., M.Y. ISCAN y E. N. L'ABBÈ. 2006. The Pattern of Vertebral Osteophyte Development in a South African Population. International Journal of Osteoarchaeology 16: 459-464.

VARELA, H. H. y J. A. COCILOVO. 2000. Structure of the prehistoric population of San Pedro de Atacama. Current Anthropology 41: 125-132.

2009. Microevolución en San Pedro de Atacama (Norte de Chile): El cementerio de Quitor. Latin American Antiquity 20:333-342.

WOO, E. y P. SCIULLI. 2013. Degenerative Joint Disease and social status in the Terminal Late Archaic Period (1000-500 B.C.) of Ohio. International Journal of Osteoarchaeology 23: 529-544.

WOOD, J. W., G. R. MILNER, H. C. HARPENDING y K. M. WEISS. 1992. The osteological paradox: problems of inferring prehistoric health from skeletal samples. Current Anthropology $33: 343-370$ 